\title{
Atrayentes de piel de tilapia, Oreochromis niloticus (Perciformes: Cichlidae), para reducir el desperdicio de alimento en granjas acuícolas
}

\author{
Carlos Roberto Pérez Reyes ${ }^{1}$ \\ 1. Núcleo Náutico Pesquero, Puntarenas, Costa Rica. Universidad de Costa Rica, Sede Regional del Pacífico. \\ Puntarenas, Costa Rica; Universidad Estatal a Distancia; cperezr@uned.ac.cr
}

\author{
Recibido 4-V-2020 • Corregido 27-VIII-2020 • Aceptado 21-IX-2020 \\ DOI: https://doi.org/10.22458/urj.v12i2.3117
}

\begin{abstract}
Attractants from tilapia skin, Oreochromis niloticus (Perciformes: Cichlidae), to reduce feed waste in aquaculture farms". Introduction: Aquaculture has emerged as an alternative to traditional fishing due to the collapse of fish populations from intensive off-shore fishing. However, many aquaculture farms could reduce feed waste by using chemical attractants to concentrate fish during feeding. Objective: To test a tilapia skin attractant. Methods: Attractants were tested with 16159 mature and immature tilapias in confined indoor freshwater and saltwater ponds, and in outdoor ponds. Results: With the attractant, the immature fish approached food faster than in the controls $(p<0.05)$. Attractants extracted from waste skin reduce pollution, are not bio-accumulable in tissue and use reagents that do not affect meat quality because they are highly soluble in water. Conclusions: The use of skin attractants is recommended as chum and not as an additive for food under controlled environments, but more research is needed in open environments.
\end{abstract}

Keywords: Chemical cues, solvent, aquaculture, fishing, methanol.
RESUMEN. Introducción: La acuicultura ha surgido como una alternativa a la pesca tradicional debido al colapso de las poblaciones de peces por la pesca intensiva en alta mar. Sin embargo, muchas granjas de acuicultura podrían reducir el desperdicio de alimento usando atrayentes químicos para concentrar los peces durante la alimentación. Objetivo: Probar un atrayente de piel de tilapia. Métodos: Se probaron atrayentes con 16159 tilapias maduras e inmaduras en estanques cerrados de agua dulce y agua salada y en estanques al aire libre. Resultados: Con el atrayente, los peces inmaduros se acercaron al alimento más rápidamente que los controles $(p<0.05)$. Los atrayentes extraídos de la piel de desecho reducen la contaminación, no son bioacumulables en los tejidos y utilizan reactivos que no afectan la calidad de la carne porque son altamente solubles en agua. Conclusiones: Se recomienda el uso de atrayentes de piel de tilapia, como cebo pero como aditivo para alimentos, en ambientes controlados. Se necesita más investigación en ambientes abiertos.

Palabras clave: Mensajeros químicos, disolvente, acuicultura, pesca, metanol.

La producción mundial de pescado, crustáceos, moluscos y otros animales acuáticos está aumentando, alcanzando los 170,9 millones de toneladas en 2016, De este total, la producción de capturas fue de 90,9 millones de toneladas, una disminución de 1,9\% en comparación con el año anterior. La producción acuícola fue de 80 millones de toneladas en 2016, 5,2 \% más que el año anterior (FAO, 2018). La actividad pesquera extractiva mantiene un descenso en las capturas por año debido a la sobrepesca (Macfadyen, Huntington, \& Cappel, 2011; Seddon, Griffiths, Soorae, \& Armstrong, 2014), situación similar se da en Costa Rica donde zonas como el Golfo de Nicoya, Golfo Dulce y la mayor parte de las zonas pesqueras están en decadencia productiva (Blanco \& Mata 1994; OSPESCA, 2011; Salas, Ross, \& Arias, 2012).

Según Wo y Moreno (2001) y Radulovich (2008), la acuicultura para sus fines, destruye ecosistemas como los manglares, los cuales son claves para la recuperación de los océanos. En la 
actualidad, todavía es una práctica muy costosa y muchos países no la consideran como una verdadera alternativa para la problemática pesquera (Arias, 2008; Otárola, 2008; Segura, 2013). Dentro del quehacer de la acuicultura, se tiene como necesidad la aplicación de alimento artificial hecho a base de harina de pescado capturado del mar. Si los organismos cultivados no se congregan adecuadamente para consumirlo de manera inmediata, se corre el riego del desperdicio de alimento, con lo que aumentan los costos operativos en la industria y la contaminación del agua (Udelhoven, 2011).

Una solución para evitar la pérdida de alimento la propone Moore (2007), él sugiere el uso de una sustancia atrayente al momento de la alimentación, los peces se congregarían en un solo punto y sería más eficiente el consumo de las proteínas brindadas. Montemayor, Badii, Flores y Aguilera (2000) agregan que el uso de estas sustancias en la acuicultura hace una pesca más eficiente por congregarse más. Las propiedades del agua de los estanques, hacen que las señales químicas sean más relevantes que las visuales, debido a la mala visibilidad para los peces (González \& Álvarez 2015).

Martin y López (2009) las definen como sustancias químicas que produce un individuo y que pueden provocar cambios en la fisiología o el comportamiento de los individuos coespecíficos. Montemayor et al. (2000) señala también que, son sustancias cuya recepción está implicada en las relaciones entre los organismos, a lo que llaman en general semioquímicas, las cuales se dividen en feromonas que opera a nivel intraespecífico y las aleloquímicas, que es entre especies. Los mensajeros químicos, por tanto, son fragancias moleculares liberadas en el agua por los peces, como una forma de comunicación y favorecen la congregación de los peces (Stacey, Chojnaeki, Narayanan, Cole, \& Murphy, 2003); el fin es favorecer las interacciones sociales, el reconocimiento de parientes, la detección de la presa y el señalamiento de depredadores (Tifner, Zanin, \& De Bórtoli, 2003; Moore, 2007, Nunes, 2013).

En pequeñas concentraciones, las señales químicas pueden ser detectables en el agua por el sistema olfativo de los peces, y su presencia en el alimento podría inducir un cambio en su comportamiento, de modo que se conglomeren y sean más agresivos en la alimentación; en concentraciones de hasta 10 moles L-1 de triptófano en atunes aleta amarilla o de 0,1mg L-1 de trucha arcoíris (Montemayor et al., 2000). Su aplicación ha permitido describir más detalladamente el comportamiento social, hasta niveles jerárquicos, en poblaciones de varias especies de peces de interés comercial en acuicultura (Botero, 2004). El uso de mensajeros químicos como atrayente en la acuicultura, ya fue probado con buenos resultados en especies como tilapia, bacalao europeo y camarón blanco usando productos sintéticos similares a las que poseen estos organismos naturalmente (Moore, 2007).

En aguas abiertas, el uso de mensajeros químicos también se ha dado con gran suceso. Un experimento con pesca de caña para capturar Salmones en Cataluña de España dio tan buenos resultados, que motivó a las autoridades la necesidad de regular la actividad por el peligro latente de sobreexplotación (Dodd \& Dodd, 2005). Por este motivo, se han establecido una serie de restricciones y reglamentos, para evitar este problema con los atrayentes en la pesca, como sucedió en España con la pesca deportiva (Montilla, 2005).

La mayor limitante en el uso de mensajeros químicos sigue siendo el difícil y lento proceso para extraer pequeñas cantidades. Por ejemplo, de las glándulas abdominales de 500000 mariposas, solo se lograron extraer $75 \mathrm{mg}$ del mensajero químico de $70 \mathrm{~g}$ de esteroides, aislados de $250 \mathrm{~g}$ de ácidos grasos (Seyhan, 1998). A pesar de este inconveniente, los animales tienden a ser muy sensibles a concentraciones homeopáticas de las mensajeras químicas en general.

La mayor cantidad de sustancias que ofrecen ser mensajeros químicos en los peces, se encuentran principalmente en su piel, según lo indica Huertas, Hubbard, Canario y Cerda (2007) en su trabajo. Su naturaleza química lo describen como alcoholes, aminoácidos y esteroides de cadena 
larga con dobles enlaces que les da la condición de ser polares (Pinillos, 2003; Tifner et al., 2003; Nunes, 2013). Esta propiedad demanda al uso de reactivos a base de alcohol, para separarlas como fue el método descrito por Huertas et al. (2007) para aislar el mensajero químico de las anguilas en una solución de $1 \mathrm{mg} \mathrm{ml}-1$ de alcohol etílico al 50\% en agua destilada. Si se logra aislar esta sustancia de manera adecuada, podrá servir de herramienta para mejorar el proceso productivo en la acuicultura, al recuperar un residuo propio como es la piel de pescado.

En la actualidad el mercado de sustancias odorantes es de origen sintético y de alto costo, dirigido especialmente a la cosmetología, militarismo y la industria alimentaria principalmente (Vizcay, 2008). Esta propuesta pretende rescatar un residuo del mismo proceso productivo para convertirlo en un atrayente orgánico de muy bajo costo. Por tanto, el objetivo de este estudio fue, probar diferentes tipos de disolventes para la extracción de mensajeros químicos orgánicos en la piel de la tilapia del Nilo (Oreochromis niloticus) con el fin de utilizarlos como un atrayente, para potenciar el rendimiento acuícola en Costa Rica.

\section{MATERIALES Y MÉTODOS}

Preparación de los extractos. La piel de tilapia, de alto grado de inocuidad, fue obtenida de una empresa exportadora de tilapia en Cañas. Las muestras fueron seleccionadas de manera aleatoria, todas procedentes de individuos maduros y que cumplen con los requisitos de comercialización $(\mathrm{N}>400 \mathrm{~g})$. El tejido estaba congelado a una temperatura de $-5^{\circ} \mathrm{C}$ y embalado en cajas de cartón totalmente limpias. Al Ilevarlas al laboratorio de la Universidad de Costa Rica en Puntarenas, se almacenaron a la misma temperatura hasta el momento de la extracción.

Para extraer los atrayentes, las pieles obtenidas de la especie se homogenizaron individualmente, por medio de maceración, con el fin de garantizar una buena mezcla. Luego, el macerado se deshidrató en una estufa y se guardó en un recipiente de vidrio sellado a temperatura ambiente. El proceso de purificación se hizo extrayendo impurezas y restos de proteínas, que conformaban la mayor parte de la masa en la mezcla de pieles secas. Para tal fin, se utilizó una solución de ácido tri cloro acético al $20 \%$, el cual reaccionó con los enlaces peptídicos de las proteínas obligándolas a precipitar (Laitinen \& Harris, 1982; Starr \& Taggart, 2004).

El resultante se disolvió en agua con hexano, como disolvente no polar, para asegurar la separación de los compuestos por su polaridad. El agua y el hexano se separaron en distintos recipientes y a cada solución acuosa se le agregó más hexano. Este proceso se repitió de 3 a 7 veces, hasta llevar la mezcla de piel a condiciones óptimas, para el posterior proceso de purificación y extracción química estaba lista para elaborar las diferentes soluciones según su concentración.

Al tener las pieles listas para su tratamiento de extracción de mensajeros químicos, se procede a ensayar la solución que mejor libere la sustancia del tejido. Para ello, se experimentaron diferentes combinaciones de agua destilada, metanol, acetonitrilo, etanol y éter etílico, todos ellos poseen una alta polaridad y alto nivel de pureza, esa fue la razón de su elección (Cuadro 1). Los extractos se mantuvieron a $5^{\circ} \mathrm{C}$ en un congelador por ser inflamables y tener alta volatilidad, hasta el día de la prueba en campo. En el momento de utilizarlos, se obtuvieron alícuotas de $30 \mathrm{ml}$ de las distintas concentraciones y se transportaron con hielo al lugar donde se realizaría la experimentación.

Se trabajó con 16159 tilapias en total utilizadas en tres ensayos específicos. El primer ensayo hace énfasis al número de ejemplares que realizan quimiotaxismo positivo ante el atrayente en el agua. El segundo ensayo analiza grupalmente el efecto del atrayente en estanques confinados bajo techo, en dos grupos: los experimentales y los de control. El tercer ensayo es similar al segundo solo que se manejan mayor cantidad de individuos y en estanques en ambientes abiertos. 


\section{CUADRO 1}

Tratamientos con distintas combinaciones de soluciones extractoras de mensajeros químicos

\begin{tabular}{clc}
\hline Tratamiento & \multicolumn{1}{c}{ Soluciones } & Proporción \\
\hline T1 & Éter etílico & 1 \\
T2 & Metanol & 1 \\
T3 & Etanol & 1 \\
T4 & Acetonitrilo & 1 \\
T5 & H2O & 1 \\
T6 & H2O+metanol & $(1: 1)$ \\
T7 & H2O+metanol y acetonitrilo & $(1: 1: 1)$ \\
T8 & Metanol+acetonitrilo & $(1: 1)$ \\
T9 & H2O+metanol y acetonitrilo & $(2: 1: 1)$ \\
T10 & H2O+metanol y acetonitrilo & $(1: 1: 2)$ \\
T11 & Metanol+acetonitrilo+H2O+etanol+éter etílico & $(1: 1: 1: 1: 1)$ \\
\hline
\end{tabular}

Ensayo 1. Las pruebas de los extractos con Tilapias se realizaron en el año 2015-2016, en la Estación Experimental Enrique Jiménez Núñez de la Universidad Técnica Nacional (UTN) en Cañas de Guanacaste, Costa Rica. Se utilizaron inicialmente individuos de tilapia maduros de $700 \mathrm{~g}(\mathrm{~N}=9)$, en una pila de concreto con obstáculos, para establecer el grado de atracción del pez hacia el mensajero químico en el agua, de tal forma que se contabilizaría el número de peces subordinados a la sustancia. La pila era de $2 \mathrm{~m}$ profundidad, $2 \mathrm{~m}$ de ancho por $3,5 \mathrm{~m}$ de largo, similar al trabajo de Reyes (2012).

Los peces se colocaban en un extremo del laberinto y en el extremo opuesto se aplicaba la sustancia al agua del estanque de manera lenta y constante, parar medir el tiempo de reacción del pez, si es que ocurría. Dicho tiempo de reacción inicia, cuando el pez responde al estímulo al momento que la sustancia entró en contacto con el agua, y finaliza cuando el pez llega al lugar donde se realizó la emisión de esta. Primero fue un pez por prueba y luego una prueba final con los nueve peces. De este trabajo se determinó la concentración más eficiente como atrayente natural. Se eliminó después de cada observación, el agua del estanque, con el fin de volver a realizar una nueva lectura. En esta primera etapa, se probaron todas las concentraciones, donde se incluían bolsas con agua destilada solamente y otra con todas las sustancias químicas sin los extractos, con 10 repeticiones todo durante dos meses (Fig.1).

Ensayo 2. En una segunda etapa, se trabajó en cuatro estanques, dos con agua salada y dos con agua dulce de plástico bajo techo, cuyas dimensiones eran Largo $(3,0 \mathrm{~m})$, ancho $(2,0 \mathrm{~m})$ y altura $(1,0 \mathrm{~m})$. En los estanques se experimentó con peces de $50 \mathrm{~g}(\mathrm{~N}=75)$ y $120 \mathrm{~g}(\mathrm{~T} 13)(\mathrm{N}=75)$ realizando 10 repeticiones con alícuotas de $30 \mathrm{ml}$ de la concentración más eficiente del ensayo 1 y $30 \mathrm{ml}$ de agua destilada como control (Cuadro 2).

En cada aplicación, al finalizar la prueba, se eliminaba el agua con la sustancia al nivel donde los peces apenas pudieran estar en suspensión, y esta se restauraba con agua fresca hasta alcanzar el nivel anterior, permitiendo la purga de agua por una hora después de alcanzado el nivel. El usar agua salada es para determinar la eficiencia de la sustancia según el grado de salinidad, dado que se espera producir esta especie en agua salada en el futuro. Posteriormente se calcula un promedio de tiempo respuesta de los controles y los tratamientos de los peces T12 y T13 según el tipo de agua en donde se encontraban. Todas las observaciones fueron a nivel grupal y no individual. 


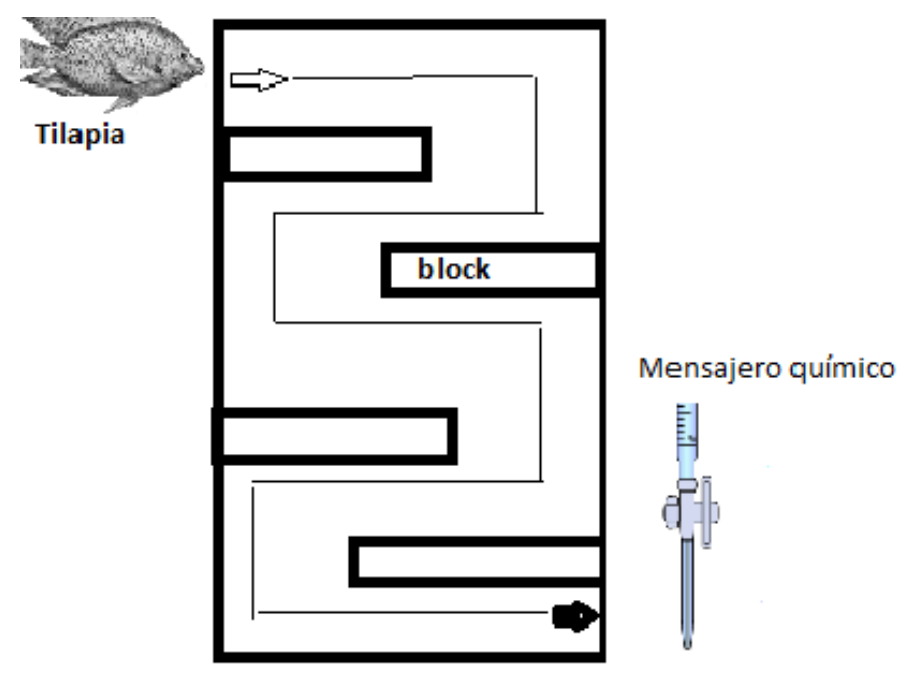

Fig. 1. Esquema del estanque de cemento con obstáculos de blocks de construcción con el fin de probar la eficiencia del atrayente.

\section{CUADRO 2}

Distribución de las Tilapias (Orechromis niloticus) ( $\mathrm{N}=150)$ según su peso $(\mathrm{g})$ en estanques de agua salada y dulce en la estación experimental de la UTN en Cañas 2016

\begin{tabular}{lcc}
\hline Tratamiento & $\begin{array}{c}\text { Estanque agua dulce } \\
\text { (peso en g) }\end{array}$ & $\begin{array}{c}\text { Estanque agua salada } \\
\text { (peso en g) }\end{array}$ \\
\hline Control & 50 & 50 \\
T12 & 50 & 50 \\
Control & 120 & 120 \\
T13 & 120 & 120 \\
\hline
\end{tabular}

Ensayo 3. En una tercera etapa, el extracto de mayor éxito se probó en cuatro estanques comerciales de tierra y de mayor tamaño, al aire libre, cuyas dimensiones eran de $100 \mathrm{~m}$ de largo, $5 \mathrm{~m}$ de ancho y $2 \mathrm{~m}$ de profundidad. Se utilizaron dos tratamientos con el extracto impregnado al alimento en cada ración diaria, en peces de $50 \mathrm{~g}(\mathrm{~N}=6000)$ y $700 \mathrm{~g}(\mathrm{~N}=10000)$ respectivamente, y dos como control sin el extracto presente, en iguales condiciones y cantidades que los estanques blancos o patrón. Se usaron las concentraciones $T 7$ y T8 en este experimento con el fin de ver eficiencia en ambientes abiertos, ya que solo se había experimentado en ambientes confinados. En cada caso se mantuvo la misma cantidad de peces con similares tamaños, donde T14 corresponde a peces inmaduros y T15 a peces maduros (Zimmermann, 2005; Saavedra, 2006; Pérez \& Sáenz, 2015). Se registró el tiempo de respuesta de los peces y se describió la congregación de estos, al momento de aplicar el alimento, se realizaron 10 repeticiones.

Finalmente, los datos obtenidos se analizaron mediante una prueba de Kruskal Wallis y $T$ student (Gutiérrez, 1995), para determinar si hubo diferencias entre los tratamientos, dado que todas las muestras provienen de una sola especie en una zona de cultivo. 


\section{RESULTADOS}

Ensayo 1. La respuesta de las Tilapias maduras en las pilas de concreto, en cuanto a cantidad y duración de la actividad, fue significativa en los tratamientos T7 y T8, de tal forma que la concentración metanol + acetonitrilo fue la más exitosa como atrayente orgánico (Kruskal-Wallis, $p<0.05)$. Los peces pudieron eludir los obstáculos y llegar rápidamente al sitio donde se había liberado la alícuota de $30 \mathrm{ml}$ de cada concentración. Cuando se contempla los nueve peces al mismo tiempo, la respuesta al estímulo en cada intento no era la misma para los peces. Un pez que llegaba de primero en una concentración no resultaba mismo en la siguiente prueba, por tanto, no estaba dirigida a un solo pez la sustancia sino al primero en recibir el estímulo biológico (Cuadro 3).

\section{CUADRO 3}

Número de tilapias que reaccionaron a la presencia de las distintas concentraciones del extracto según tiempo de respuesta $(\mathrm{N}=9)$

\begin{tabular}{ccc} 
Tratamiento & Promedio de peces & $\begin{array}{c}\text { Promedio del tiempo de } \\
\text { reacción (min) }\end{array}$ \\
\hline T1 & $0 \pm 2,316$ & $0 \pm 01: 50$ \\
T2 & $4 \pm 2,316$ & $01: 22 \pm 01: 50$ \\
T3 & $0 \pm 2,316$ & $0 \pm 01: 50$ \\
T4 & $0 \pm 2,316$ & $0 \pm 01: 50$ \\
T5 & $0 \pm 2,316$ & $0 \pm 01: 50$ \\
T6 & $0 \pm 2,316$ & $0 \pm 01: 50$ \\
T7 & $7 \pm 2,316$ & $05: 00 \pm 01: 50$ \\
T8 & $4 \pm 2,316$ & $05: 00 \pm 01: 50$ \\
T9 & $1 \pm 2,316$ & $02: 30 \pm 01: 50$ \\
T10 & $2 \pm 2,316$ & $01: 40 \pm 01: 50$ \\
T11 & $2 \pm 2,316$ & $02: 24 \pm 01: 50$ \\
\hline
\end{tabular}

Ensayo 2. Se determinó que indiferentemente si el agua era dulce o salada, los movimientos fueron los mismos, dirigidos hacia donde estaba la sustancia, de manera coordinada entre los alevines y no tanto en los adultos, los cuales se dirigían con movimientos distintos entre ellos, por tanto, no hubo diferencias significativas (T student, $p>0.05$ ). Fue el tratamiento T13 mostró una reacción inmediata que su control y el tratamiento T12, lo cual corresponde a especie de segunda madurez sexual (Cuadro 4).

\section{CUADRO 4}

Número de ejemplares que observaron reacción positiva a las mezclas, a distintas concentraciones Metanol + Acetonitrilo según tiempo de respuesta $(N=150)$

\begin{tabular}{ccccc}
\hline Tratamientos & Estanques & Peso $(\mathbf{g})$ & Condición & $\begin{array}{c}\text { Tiempo promedio en 10 } \\
\text { repeticiones (min) }\end{array}$ \\
\hline T12 & Dulce y salado & 50 & Inmaduro & $00: 30$ \\
Control & Dulce y salado & 60 & Inmaduro & $00: 00$ \\
T13 & Dulce y salado & 700 & Maduro & $01: 10$ \\
Control & Dulce y salado & 700 & Maduro & $00: 00$ \\
\hline
\end{tabular}


Ensayo 3. La concentración Acetonitrilo+ metanol + agua destilada, los grupos de peces experimentales obtuvieron mejor tiempo de respuesta que los peces del grupo control, mientras que la concentración Acetonitrilo + Metanol, tuvo mejor tiempo de respuesta los peces maduros del grupo control (Kruskal-Wallis, $p<0.05$ ). En el caso de los inmaduros, no tuvo diferencias significativas entre el control y la concentración de Acetonitrilo+ metanol + agua (Kruskal-Wallis, $p>0.05)$, mientras que los alevines tuvieron mejor tiempo de respuesta con la concentración Acetonitrilo + metanol, donde en 30s se tuvo una reacción del alimento impregnado con la sustancia, sin embargo, no discrimina el control ya que, en los intentos en promedio, se tuvo resultados muy similares (Cuadro 5).

\section{CUADRO 5}

Tiempo de reacción de Tilapias maduras e inmaduras en estanques abiertos con el uso de alimentos impregnados con el mensajero químico en las concentraciones Acetonitrilo+ metanol + Agua y Acetonitrilo + Metanol

\begin{tabular}{cccccc}
\hline Tratamiento & $\begin{array}{c}\text { Peso del } \\
\text { pez }(\mathbf{g})\end{array}$ & $\begin{array}{c}\text { Condición del } \\
\text { pez }\end{array}$ & $\begin{array}{c}\text { Peso de piel } \\
\text { seca }(\mathbf{g})\end{array}$ & $\begin{array}{c}\text { Número de } \\
\text { peces }\end{array}$ & $\begin{array}{c}\text { Tiempo de respuesta } \\
\text { (min) }\end{array}$ \\
\hline T15 & 700 & Maduro & 143,13 & 3000 & $01: 10$ \\
T14 & 50 & Inmaduro & 143,2 & 3000 & $01: 10$ \\
Control & 700 & Maduro & 0 & 10000 & $01: 30$ \\
T14 & 50 & Inmaduro & 143 & 6000 & $00: 30$ \\
T15 & 143 & Maduro & 143 & 8000 & $01: 10$ \\
Control & 60 & Inmaduro & 0 & 10000 & $00: 30$ \\
\hline
\end{tabular}

\section{DISCUSIÓN}

Extractos. Según el trabajo realizado, el metanol y el acetonitrilo fueron las sustancias que mejor extraen el mensajero químico. El metanol es una disolvente muy usado en la síntesis y purificación de sustancias orgánicas, por ejemplo, Huertas et al. (2007) usaron el metanol y agua destilada para extraer mensajeros químicos del mucus de la piel de anguilas europeas con muy buenos resultados. De igual forma, la sustancia acetonitrilo es un disolvente de polaridad media, que tiene una excelente miscibilidad con el agua y de una aceptable afinidad líquida con otras sustancias. Su baja reactividad química, la convierten en una sustancia muy popular en procesos de extracción (Laitinen \& Harris, 1982; Chang, 2008; Chang \& Goldsby 2014, Sammeer \& Kanakapura, 2011).

Ambos compuestos, el metanol y el acetonitrilo, no son bioacumulables en tejidos de seres vivos, y pueden descartarse sin problema al ambiente, debido a su alta volatilidad y solubilidad. Lo anterior se demuestra en un estudio realizado con la extracción de mensajeros químicos en la piel del pez Lepomis macrohirus (mojarra azul) en América del Norte, donde se demostró que los tejidos de los peces experimentales no evidenciaron el fenómeno de bioacumulación en su carne (MERK, 2016). Es importante puntualizar que en este trabajo la bioacumulación no fue el objetivo principal, por lo que es importante hacer análisis de inocuidad a los peces tratados con estos extractos en el futuro.

Ensayos. Los peces inmaduros responden de manera más rápida a los atrayentes que los adultos, pero ambos, son atraídos por la sustancia sin importar si es en agua dulce o agua salada. Gómez, Padilla, López, Núñez y Fernández (2014) indican en su estudio que la madurez sexual está dada a partir de los $200 \mathrm{~g}$ en adelante, a pesar de que en su mismo trabajo señalan que en la literatura, la primera madurez de Oreochromis niloticus se encuentra en el rango de los 70 a $100 \mathrm{~g}$ aproximadamente, ello explicaría su mayor sensibilidad ante la sustancia. 
Las glándulas de piel no están sometidas por las hormonas sexuales, sino a la respuesta a los cambios inmediatos del medio circundante, puesto que ha sido fijada como parte indispensable de su supervivencia. La comunicación química en peces es parte de un proceso evolutivo, primero existían individuos que liberaban mensajeros químicos que no eran detectables por sus semejantes y otras especies, luego algunos individuos comenzaron a relacionar el olor de la sustancia con algún evento o suceso, de modo que iniciaron una serie de respuestas ante su presencia en el medio (Heras, 2014). Finalmente, se da una especiación, donde los organismos de la misma especie e inclusive de distintas, se comunican por medio de mensajeros químicos (Stacey et al., 2003).

Lo anterior no implica que los mensajeros químicos provenientes de la piel, no tengan una función reproductiva. Según Peña (2009) y Heras (2014) los mensajeros químicos de piel dan una selección positiva para el aislamiento reproductivo, y esto se evidencia en Tilapias, donde los machos al liberar el mensajero químico, inician seguidamente la copula y no antes. Claro está, las sustancias de comunicación química pueden provenir de otros órganos, como los riñones y estar presentes en la orina también. En este trabajo solo se realizó con la piel de pez y una nueva propuesta podría ser trabajar con la extracción en otros órganos para determinar eficiencia de los atrayentes.

Los extractos provenientes de la piel de los peces, mostraron ser un buen atrayente por la presencia de mensajeros químicos, que son rápidamente detectables en el proceso de alimentación. Esto obedece a la presencia de aminoácidos en el mucus de la piel, podría considerarse la base de las sustancias atrayentes. Al estar en contacto con el agua, la piel puede liberar mensajeros químicos a una tasa mayor que el resto de los órganos del cuerpo para ser recibido por otro pez (Stacey et al., 2003; Heras, 2014). No obstante, se debe considerar para la extracción de estos atrayentes en peces que provienen de ambientes abiertos, el enhielado y almacenamiento con otras especies, ya que puede mezclar mensajeros químicos de otras especies, lo cual puede producir un error experimental.

A parte de la capacidad de disolución, está la manera de aplicación del mensajero químico. Según Moore (2007) es recomendable que estas no se introduzcan como un aditivo en los alimentos, sino que se agreguen mediante el rocío sobre el alimento de 2 a 5 minutos antes de la alimentación, simulando la emisión en piel de la sustancia atrayente. Esto permite que la detección de los mensajeros químicos por parte de los peces, sea más rápida y se congreguen en mayor cantidad a la hora de alimentarse (Nunes, 2013). Es importante tener presente que, las sustancias provienen de la piel y que al momento de alimentarse se debe recrear este proceso.

El órgano que recibe estas señales químicas está relacionado al sentido del olfato, el cual es capaz de detectar y segregar compuestos orgánicos volátiles y de bajo peso molecular (Vizcay, 2008). Se divide en sistema olfatorio principal compuesto por: cavidad nasal, bulbo y epitelio olfatorios. Dentro del bulbo olfatorio se encuentra el órgano vomeronasal y los nervios vomeronasal, esta es la clave para que los peces interpreten el mensaje. En general el sistema accesorio se especializa en detección de feromonas, mientras que el sistema principal detecta olores comunes (García, 2013).

La presencia de un mensajero químico, por tanto, produce cambios a nivel celular, principalmente en las neuronas sensoriales cuyos receptores específicos detectan el estímulo a nivel de membrana celular y mediante una vía sináptica llega al hipotálamo, lo que, de forma inconsciente incluso, le da la capacidad al pez de detectar la señal química y reaccionar casi de forma inmediata al estar en contacto (Bubis, 2003; Tifner et al., 2003; Vizcay, 2008). Este proceso evolutivo les ha permitido a los peces vivir de manera grupal, para localizar alimento y detectar depredadores, pero lo más relevante es que funciona mejor en individuos fenotípicamente similares (González \& Álvarez, 2015). Ello explica el comportamiento observado tanto en estanques confinados como los 
de áreas abiertas. Los peces reaccionaban de inmediato a la presencia de la sustancia de origen orgánica.

Finalmente, este trabajo demostró que la extracción es sencilla y genera un atrayente cuyo efecto es el agrupamiento de los peces en acuicultura, y que eventualmente funciona para congregar los peces para alimentación y salud. El estudio demostró una mejor respuesta en el tiempo por parte de los peces experimentales que los peces del grupo control; sin embargo, se debe investigar más en el tema. La congregación mejora la salud de los peces, porque se alimentan mejor y los hace más resistentes a las enfermedades al ser más agresivos durante este proceso. Experiencias descritas por Moore (2007), indican que la alimentación usando mensajeros químicos sintéticas, propiciaron un mayor crecimiento de Tilapias, llegando a un $17 \%$ con respecto a las que se encontraban en estanques de control y esto lo confirma Mioso, Toledo, Bravo y Bessonart (2014) en su estudio, aunque en ningún momento utilizaron atrayentes de origen orgánico.

Se recomienda como una segunda etapa, caracterizar molecularmente los mensajeros químicos de especies populares en acuicultura y otras como el Pez León, una especie plaga en el Caribe que se debe erradicar, y con este método podría ayudar en el control de la población. Lo anterior se lograría, al tener un mejor detalle de su estructura molecular y composición, ya que se podrá aislar y purificar con el fin de tener una mejor eficiencia en las granjas acuícolas. Con estos trabajos la pesca extractiva dará paso a la acuicultura por ser más rentable en el tiempo por su eficiencia y eficacia.

\section{AGRADECIMIENTOS}

Se agradece a Marietta Villalobos, Marvin Castro, Marjorie Jiménez y Johnny Aguilar por su orientación y consejos al proyecto. A las profesionales en Química, Mariela Araya y Joyce Castro de la Escuela de Química de la Universidad de Costa Rica, por su aporte en las técnicas de extracción de la sustancia. A William Alvarado de la Universidad Técnica Nacional de Cañas, por ceder sin ningún costo, las instalaciones para llevar a cabo los experimentos con tilapia. A Martín Méndez, por facilitarnos piel de tilapia a ningún costo para este proyecto en la empresa donde labora. A Freddy Zamora por la revisión del inglés en este trabajo, del Núcleo Náutico Pesquero del Instituto Nacional de Aprendizaje y a Ericka González por la revisión de la redacción del documento. A Karla Pérez Chaverri quien ayudó en la experimentación en los estanques y la extracción del mensajero químico en el laboratorio.

\section{ÉTICA, CONFLICTO DE INTERESES Y DECLARACIÓN DE FINANCIAMIENTO}

El autor declara haber cumplido con todos los requisitos éticos y legales pertinentes, tanto durante el estudio como en el manuscrito; que no hay conflictos de interés de ningún tipo, y que todas las fuentes financieras se detallan plena y claramente en la sección de agradecimientos. Asimismo, están de acuerdo con la versión editada final del documento. El respectivo documento legal firmado se encuentra en los archivos de la revista.

C.P.R. realizó todas las actividades de esta investigación y se declara único autor de la obra. 


\section{REFERENCIAS}

Arias, S. (2008). Pobreza en las costas ticas y potencial de la acuicultura. Revista Ambientico, 179(19), 18-19.

Blanco, O., \& Mata, A. (1994). La Cuenca del Golfo de Nicoya: un reto al desarrollo sostenible. San José, Costa Rica: Editorial Universidad de Costa Rica.

Botero, M. (2004). Comportamiento de los peces en la búsqueda y la captura del alimento. Revista Colombiana de Ciencias Pecuarias, 17(1), 63-75.

Bubis, J. (2003). ¿Cómo olemos? Academia Biomédica Digital, (15), 21.

Chang, R. (2008). General Chemistry. NY, EEUU: McGraw Hill.

Chang, R., \& Goldsby, K. (2014). General Chemistry. NY, EEUU: McGraw Hill.

Dodd, G., \& Dodd, H. (2005). Composición de una feromona. Oficina Española de Patentes y Marcas. № ES 2239403 T3, 7. España.

FAO. (2018). Estadística de pesca y acuicultura del año 2016. Roma, Italia: Departamento de Pesca y Acuicultura de la FAO.

García. P. (2013). Expresión de c-fos en vermis cerebelar de ratas macho, por estimulación olfativa, durante la adquisición de experiencia en la conducta sexual (Tesis de Maestría). Universidad Veracruzana, Instituto de Neuroetología, Xalapa, México.

González, C., \& Álvarez, D. (2015). La conducta de los peces como bioindicadores de la presencia de estresores ambientales. Campeche, México: ECOSUR \& Instituto Nacional de Ecología y Cambio Climático.

Gómez, M., Padilla, C., López, M., Núñez, G., \& Fernández, L. (2014). Maturity size and fecundity of hybrid tilapia Oreochromis aureus X Oreochromis niloticus (Perciformes: Cichlidae) from the reservoir Fernando Hiriart Balderrama "Zimapan", Hidalgo, Mexico. UNED Research Journal, 6(2), 169-179.

Gutiérrez, E. (1995). Métodos estadísticos para las ciencias biológicas. Heredia, Costa Rica: EUNA.

Heras, J. (2014). Adaptive molecular evolution within a highly diverse group of fishes, rockfishes (Thesis degre of Doctor of Philosophy). University of California, Quantitative and Systems Biology, California, EEUU. Recuperado de https://escholarship.org/content/qt88d3q7ws/qt88d3q7ws.pdf

Huertas, M., Hubbard, P., Canario, A., \& Cerda, J. (2007). Olfactory sensitivity to conspecific bile fluid and skin mucus in the European eel Anguilla anguilla (L.). Journal of Fisch Biology, 70, 1907-1920.

Laitinen, H., \& Harris, W. (1982). Análisis químico: texto avanzado y de referencia. Barcelona, España: Reverte.

Macfadyen, G., Huntington, T., \& Cappel, R. (2011). Aparejos de pesca abandonados, perdidos o descarados. Roma, Italia: FAO-PNUMA.

Martí-n, J., \& López, P. (2009). Evolución de señales químicas en los procesos de selección sexual en reptiles. En Dopazo, H. \& Navarro, A. (Eds.) Adaptación y Evolución. 150 Años Después del Origen de las Especies (pp.261-269). Sociedad Española de Biología Evolutiva. Valencia, España: Ed. Obra Propia.

MERK. (2016.). Propiedades químicas de acetonitrilo y metanol. Recuperado de http://www.merckmillipore.com/INTERSHOP/web/WFS/Merck-GT-Site/es_ES/-/GTQ/ProcessMSDSStart?PlainSKU=MDA_CHEM-106018\&Origin=PDP

Mioso, R., Toledo, F., Bravo, I., \& Bessonart, M. (2014). Química de productos naturales aplicada a la acuicultura: una revisión interdisciplinar. Química Nova, 37(3), 513-520.

Montemayor, J., Badii, M., Flores, A., \& Aguilera, C. (2000). La comunicación química de los organismos. Biomedicina y biología experimental, 1(2), 65-69. 
Montilla, J. (2005). Ordenación sostenible de la pesca en aguas continentales. Diario Oficial de la Generalidad de Cataluña, $5.5536,29$.

Moore, A. (2007). Feromonas como atracantes en la alimentación para la acuicultura sostenible sostenible. Ponencia presentada en la Conferencia de Aquafeed.com (pp 04-08): Londres, United Kindom.

Nunes, A. (2013). Reponses of tilapia (Oreochromis mossambicus) females to male pheromones (Tesis Grau de Mestre). Universidade do Albarve. Faculdade de Ciencias e Tecnologías, Faro, Portugal.

OSPESCA. (2011). Código de ética para la pesca y acuicultura responsable en los estados del istmo Centroamericano. San Salvador, El Salvador: FAO.

Otárola, Á. (2008). Producción acuícola continental en Costa Rica. Revista Ambientico, 179(19), 3-6.

Peña, M. (2009). Piel de la tilapia y cachama, la mina del colágeno. Recuperado de http://www.vanguardia.com/historico/31997-piel-de-la-tilapia-y-cachama-la-mina-del-colageno

Perez, M \& Sáenz, M. (2015). Crecimiento de las Tilapias Oreochromis niloticus en cultivo monosexual y ambos sexos, en sistemas de producción semi intensivos (Tesis de Ingeniería). Universidad Autónoma de Nicaragua, Facultada de Ciencias y Tecnología, León, Nicaragua.

Pinillos, M. (2003). Esteroides sexuales en Ciprinos, ciclo estacional, dinámica de liberación al agua, afinidad por las proteínas plasmáticas y sensibilidad olfatoria en la tenca (Tinca tinca, L.) (Tesis doctoral). Universidad Complutemnse de Madrid, Madrid, España.

Radulovich, R. (2008). Maricultura en mar abierto en Costa Rica. Revista Ambientico, 179(19), 7-14.

Reyes, F. (2012). Comunicación química asociada al comportamiento sexual en Austrolebias reicherti (Cyprinodontiformes, Rivulidae) (Tesis de Licenciatura). Universidad de la República Montevideo, Montevideo, Uruguay. Recuperado de https://www.colibri.udelar.edu.uy/jspui/bitstream/20.500.12008/1468/1/uy24-16068.pdf

Saavedra, M. (2006). Manejo del cultivo de tilapia. Nicaragua: BIDEAUSAID. Recuperado de https://www.crc.uri.edu/download/MANEJO-DEL-CULTIVO-DE-TILAPIA-CIDEA.pdf

Salas, E., Ross, E., \& Arias, A. (2012). Diagnóstico de áreas marinas protegidas áreas marina para la pesca responsable en el Pacifico costarricense. San José, Costa Rica: Fundación MarViva.

Sameer A. M., \& Kanakapura. B. (2011). Sensitive and Selective Spectrophotometric Determination of Gabapentin in Capsules Using Two Nitrophenols as Chromogenic Agents. International Journal of Analytical Chemistry, 1, 1-9 DOI: $10.1155 / 2011 / 619310$

Seddon, P., Griffiths, C., Soorae, P., \& Armstrong, D. (2014). Reversing defaunatoin: Restoring species in a changin world. Science, 345(6195), 406-412.

Segura, O. (2013). Pesca y acuicultura en el contexto del desarrollo social y económico de Costa Rica y Centroamérica. Trabajo presentado en el Ministerio de Trabajo, Conferencia sobre la Pesca y acuicultura, San José, Costa Rica.

Seyhan, E. (1998). Química Orgánica: estructura y reactividad. Barcelona, España: REVERTE.

Stacey, N., Chojnaeki, A., Narayanan, A., Cole, T., \& Murphy, C. (2003). Hormonally derived sex pheromones in fish: exogenous cues and signals from gonad to brain. Canadian Journal of Physiology and Pharmacology, 81, 329341.

Starr, C., \& Taggart, R. (2004). Biología: La unidad y la diversidad de la vida. New York, USA: Thompson. 
Tifner, S., Zanin, L., \& De Bórtoli, M. (2003). Efecto de la feromona androsterona sobre el estado anímico en una muestra de mujeres. International Journal of Clinical and Health Psychology, 3(1), 77-87.

Udelhoven, J. (2011). Factibilidad de los acuerdos de conservación marina. Seattle-Washington, USA: The Nature Conservancy.

Viscay, (2008). Clasificación de estímulos de odorantes mediante señales biológicas (Tesis de Maestría). Universidad de Chile. Instituto de Ciencias Físicas y Matemáticas, Santiago de Chile, Chile.

Wo, E., \& Moreno, M. (2001). Evaluación nacional sobre las fuentes de contaminación y actividades humanas originadas en Tierra que afectan los ambientes marinos costeros y dulceacuícolas asociados. San José, Costa Rica: CODARENA

Zimmermann, S. (2005). Reproducción de Tilapias. En Daza, P., Landines, M., Sanabria, A.I. (Eds.). Reproducción de peces en el trópico. (pp. 147-164). Bogota, Colombia: Instituto Colombiano de Desarrollo Rural (INCODER). Imprensa Nacional de Colombia. Recuperado

https://www.researchgate.net/publication/272813075_Reproduccion_de_Tilapias de 